\title{
REVIEW - OBSERVATIONS OF RECENT COMETS - ION TAILS
}

John C. Brandt

In this review, we concentrate on an aspect of the physics of ion tails stimulated by observations of Comet Kohoutek (1973f), namely, the nature of moving structures in the tail. These motions could be bulk motions themselves or waves moving at the information speed of the medium; the resolution of this question from simple photographic data alone may not be possible.

We do not discuss the principal problem of ion tails, namely, the physical mechanism responsible for the creation of $\mathrm{CO}^{+}$ions. Indeed, results from studies of gas phase chemistry presented at this Colloquium indicate that the solution may be quite complex. Also, we do not discuss either the details of the solar-wind comet interaction or dust tails.

An extensive series of plates of Comet Kohoutek was obtained at the Joint Observatory for Cometary Research (JOCR) with a Schmidt camera expressly designed for large-scale photography of tails. An $8^{\circ} \times 10^{\circ}$ field is recorded on $4^{\prime \prime} \times 5^{\prime \prime}$ plates or film. The JOCR plates were also a major part of the movie of Comet Kohoutek prepared by Jockers, Roosen, and Cruikshank and shown at this Colloquium. The features under discussion are best seen in movies.

Jockers, Roosen, and Cruikshank have studied the movie of Comet Kohoutek and conclude that the pattern speeds are essentially the speeds of material motion along the tail. This is the generally prevailing view and has been presented in some detail by Jockers, Lüst, and Nowak (1972) for the case of Comet Tago-Sato-Kosaka. 
An alternate view has been suggested by Hyder, Brandt, and Roosen (1974) in a preliminary discussion of the JOCR plates*. We draw heavily on this paper in the following discussion and use structures observed on one day as an example.

On January 13, 1974, photographs of Comet Kohoutek showed an (apparently) helical structure (Figure 1) which moved down the tail at approximately $200 \mathrm{~km} / \mathrm{sec}$ (the observed speed was approximately $250 \mathrm{~km} / \mathrm{sec}$ and the apparent geocentric speed of the comet was approximately $50 \mathrm{~km} / \mathrm{sec}$ ) as illustrated schematically in Figure 2. Circumstances of the observations are: $r=0.58$ a.u., $\delta=0.81$ a.u., and $\csc \beta=1.00$ (i.e., the tail was nearly face-on). The helix was approximately $16 \times 10^{6} \mathrm{~km}$ from the nucleus and had wavelength and radius of $1.4 \times 10^{6} \mathrm{~km}$ and $2.3 \times 10^{5} \mathrm{~km}$, respectively. The length of the helical structure sketched in Figure 2 is $3.6 \times 10^{6} \mathrm{~km}$.

Hyder et al. (1974) have suggested that this structure might be the result of a "kink instability" resulting from currents flowing along the tail axis. On their interpretation, the phase speed would be the Alfvén speed in the cometary plasma. This interpretation implies a cometary field of roughly 100\%. If the field configuration is essentially cylindrical away from the head, the decrease in density of $\mathrm{CO}^{+}$ions from $\sim 10^{3} \mathrm{~cm}^{-3}$ near the head to $\sim 10 \mathrm{~cm}^{-3}$ well into the tail could produce a variation in Alfvén speed from $\sim 20 \mathrm{~km} / \mathrm{sec}$ to $\sim 200 \mathrm{~km} / \mathrm{sec}$, and allow the extreme view that most moving features observed in cometary ion tails are waves moving at the Alfvén speed (see Ness and Donn 1966).

These divergent viewpoints for the case of Comet Kohoutek are based to a large extent on the same observational material. Hence, tests of the two hypotheses not based on simple, direct photography would be most desirable.

\footnotetext{
*Full publication of the plates will be in "The JOCR Atlas of Comet Kohoutek" (Roosen and Brandt, in preparation).
} 


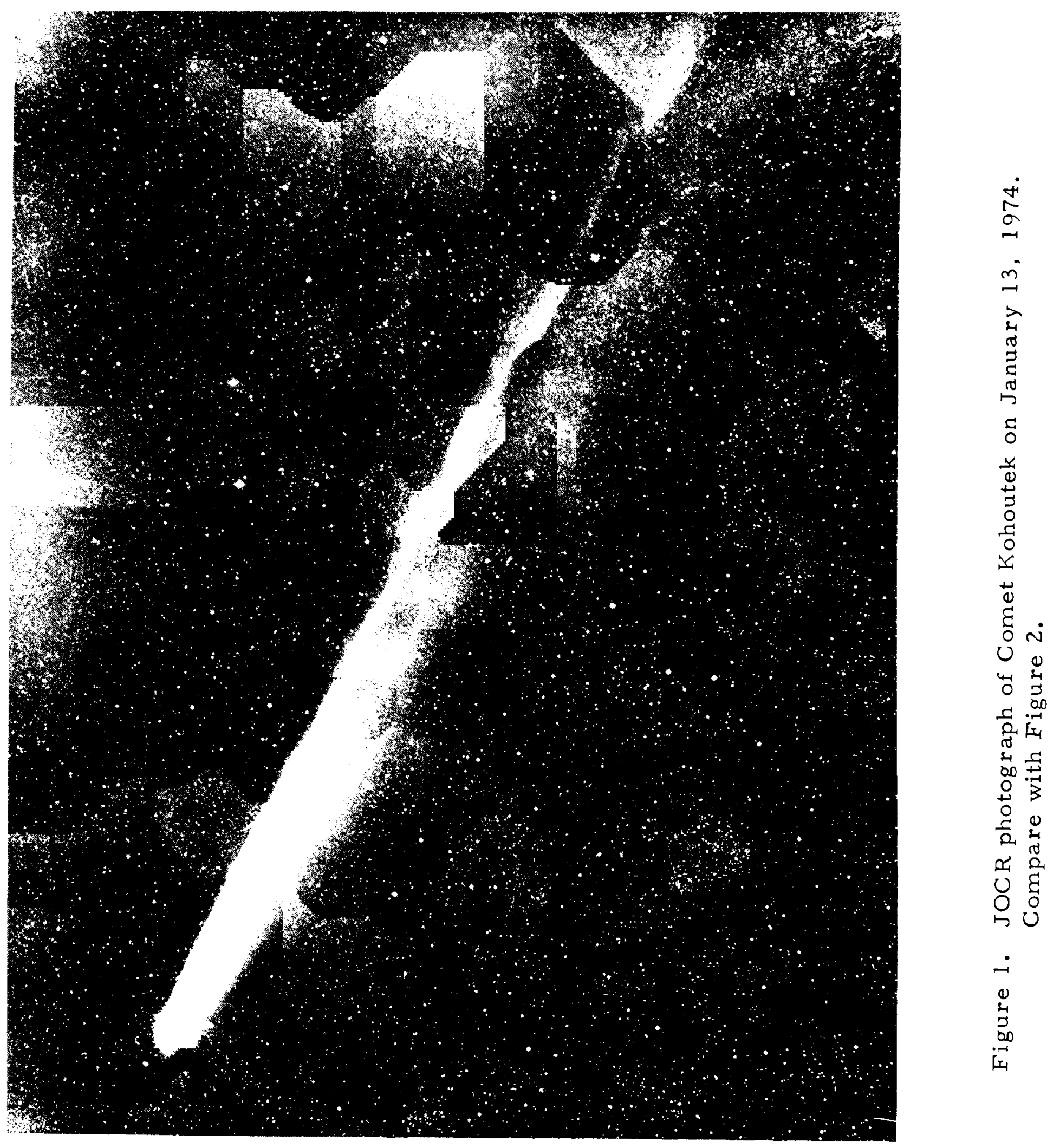




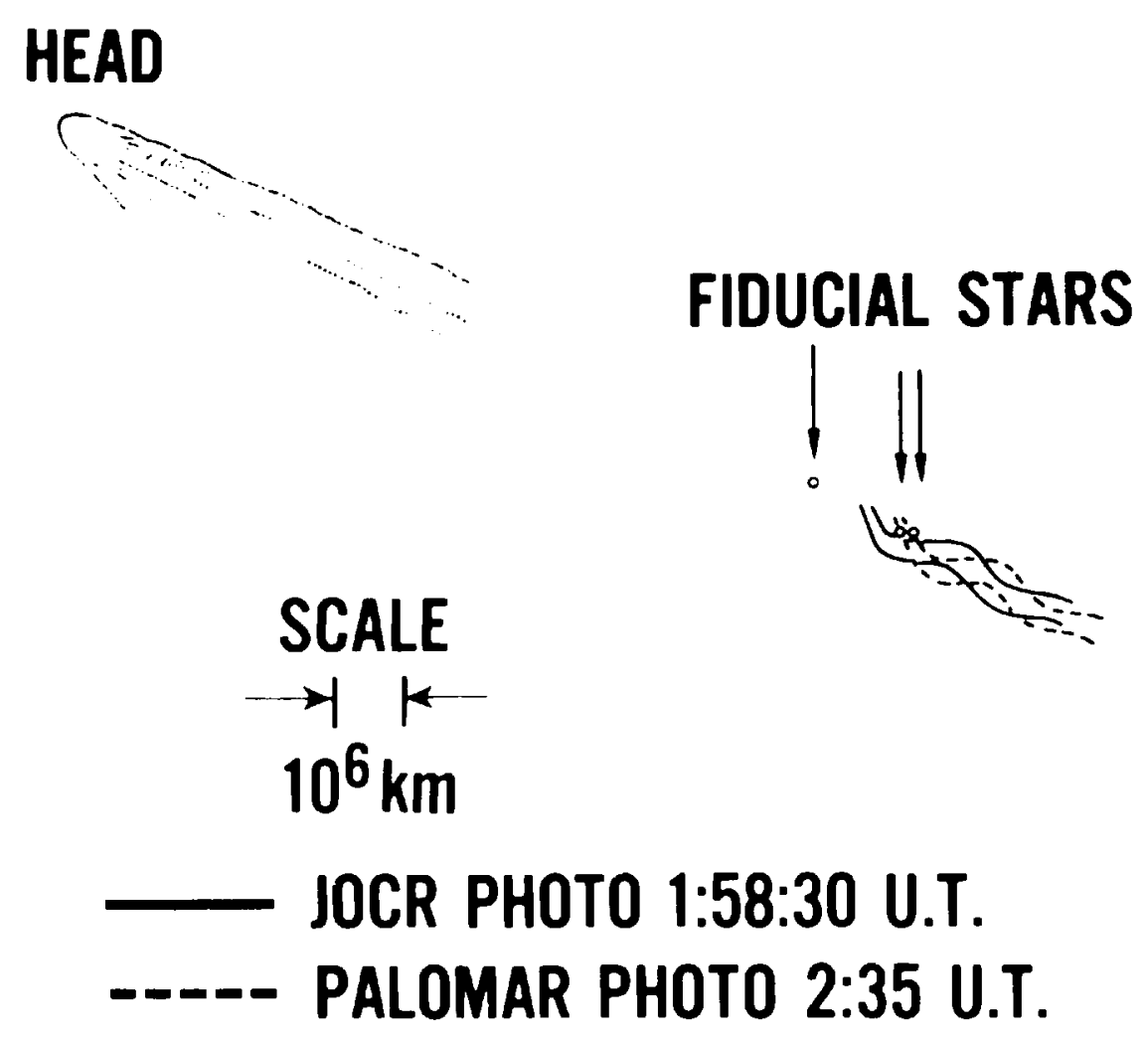

Figure 2. Schematic diagram showing the helical structure on January 13, 1974 (Figure 1) and its motion.

The most convincing test would be to observe the motion of the material spectrosocopically by means of the doppler effect. This might be carried out in two ways. (1) The material speed could be determined by observations made at a small angle with respect to the tail axis and compared with the pattern speeds. Such observations have been discussed many times but, to this reviewer's knowledge, have never been successfully carried out. Observations nearly perpendicular to the tail axis could also provide a test as suggested by Hyder (1974, private communication). If the structure seen in Comet Kohoutek on January 13, 1974 is in fact a helical wave moving much faster than the material speed, then during the passage of one wavelength of the pattern the material must traverse a circle of radius $\mathrm{r}=2.3 \times 10^{5} \mathrm{~km}$ in approximately $7 \times 10^{3} \mathrm{sec}$; see above for input numbers. 
be the Alfvén speed in the cometary plasma. This interpretation implies a cometary field of roughly $100 \%$. If the field configuration is essentially cylindrical away from the head, the decrease in density of $\mathrm{Co}^{+}$ions from $-10^{3} \mathrm{~cm}^{-3}$ near the head to $-10 \mathrm{~cm}^{-3}$ we11 into the tail could produce a variation in Alfvén speed from $\sim 20 \mathrm{~km} / \mathrm{sec}$ to $\sim 200 \mathrm{~km} / \mathrm{sec}$, and allow the extreme view that most moving features observed in cometary ion tails are waves moving at the Alfvén speed (see Ness and Donn 1966).

These divergent viewpoints for the case of Comet Kohoutek are based to a large extent on the same observational material. Hence, tests of the two hypotheses not based on simple, direct photography would be most desirable.

The most convincing test would be to observe the motion of the material spectroscopically by means of the doppler effect. This might be carried out in two ways. (1) The material speed could be determined by observations made at a sma11 angle with respect to the tail axis and compared with the pattern speeds. Such observations have been discussed many times but, to this reviewer's knowledge, have never been successfully carried out. (2) Observations nearly perpendicular to the tail axis could also provide a test as suggested by Hyder (1974, private communication)。 If the structure seen in Comet Kohoutek on January 13, 1974 is in fact a helical wave moving much faster than the material speed, then during the passage of one wavelength of the pattern the material must traverse a circle of radius $\mathrm{r}=2.3 \times 10^{5} \mathrm{~km}$ in approximately $7 \times 10^{3} \mathrm{sec}$; see above for input numbers. Thus, the material in the helix is performing 
circular motion at approximately $200 \mathrm{~km} / \mathrm{sec}$ and speeds of this size should be observed at the crests and troughs of the pattern. Very Iittle motion perpendicular to the axis of the tail would be expected if the speed of the material is about the same as the pattern speed. Thus, observations perpendicular to the tail axis may provide a clear distinction between the two viewpoints. Nearly perpendicular viewing conditions may be fairly common and were available for Comet Kohoutek for several days in mid-January 1974. Photography through shiftable, high efficiency, narrow-band filters may be the best method of observation. Speeds of $200 \mathrm{~km} / \mathrm{sec}$ would produce shifts of nearly $3 \AA$ in the blue $\mathrm{CO}^{+}$ bands, and hence, the filters needed should not be difficult to obtain. Hence, the problem reduces to finding a line of sufficient strength and suitable isolation in (say) the $A^{2} \pi-x^{2} \Sigma$ (comet tail system) bands of $\mathrm{CO}^{+}$.

\section{REFERENCES}

Hyder, C. L., Brandt, J. C., and Roosen, R. G. 1974, Icarus, in press (The Kohoutek Issue).

Jockers, K., Lüst, Rh., and Nowak, Th. 1972, Astron. and Astrophys., $21,199$.

Ness, N. F., and Donn, B. D. 1966, Nature et Origine des Comètes, (Liège), p. 343 . 


\section{DISCUSSION}

D. J. Malaise: This is surely an impressive way of showing the variability of tail features, but it is not easy to follow up the details, because it is going too fast. You repeated each image twice. To my knowledge, this is the standard in high quality cartoons (Walt Disney, for instance), but repeating each picture four times does not change the quality of the motion picture by an amount appreciable to the eye. This factor of two in the speed of motion would certainly make the show more comfortable to follow. Another point is that if the motion of the comet could be taken out, it would be much easier to follow the structure change, even if the stars are shooting back and forth.

K. Jockers: There is a repetition of each sequence 20 times. In the sequence every picture is shown twice, and sometimes there are small time gaps, so that two pictures are separated not by ten but by fifteen minutes. Then the picture is repeated three or four times.

F. Scherb: How did you separate the Alfven wave speed from the speed of the underlying plasma which is streaming outward? The apparent motion of the wave to the observer is the result of the wave motion through the tail plasma at the Alfven speed plus the motion of the tail plasma.

J. C. Brandt: We calculated the Alfven velocity would be the same as the pattern speed, and we have the pattern speed to within $10 \%$.

K. Jockers: How do ions not detectable in visible light contribute to the density needed to evaluate the Alfvén speed?

J. C. Brandt: We took the density to be solely, for the purposes of calculation, what it is for $\mathrm{CO}_{+}$. I'll simply point out that it is not terribly sensitive to that, because it appears as the square root unless we're just completely mistaken.

K. Jockers: In the cometary movie we see secondary tail rays emerging from tail condensations and moving through the main tail. If Alfven's model of cometary tail rays is correct, that means that magnetic field lines generate in the whole tail and get hooked up at condensations. So one would expect that a comet tail is not a more or less homogeneously magnitized rod but has a rather complicated magnetic field structure.

J. C. Brandt: I would certainly argue that the magnetic field model is not complicated. On the other hand, one has to start somewhere, and we can get some insight as to what the tail configuration might be by looking, for example, at the geomagnetic tail, where a cylindrical model is not perfect, but it's not 


\section{DISCUSSION (Continued)}

unreasonable, either. The field strength in the geomagnetic tail decreases very slowly-I think it is the cube root of the distance, or something like that-so that is not an unreasonable approximation.

We spent a great deal of time looking rather hard at a lot of days in which the field is fairly quiet, and the cross section does not seem to change terribly much going from fairly close to the head to a more distant part of the tail, obviously excluding the region in the immediate vicinity of the head, where a great deal of activity is going on. That's why I prefaced this discussion by mentioning that this clearly is very simple, and very simpleminded. But I have not yet seen anything of this nature that's been discussed extensively in the literature, with exceptions here and there.

L. Biermann: As I described in the paper I referred to yesterday, the visible ions are rather likely to be transformed by ion-molecule reactions into other invisible ions before being eliminated by dissociation, recombination, or complete decomposition. In any case, the visible cometary ions give only a lower limit to the actual density, though the total density may not be more than several times larger.

B. Donn: The important and impressive feature of the paper we have just heard is that Brandt and his colleagues and Jockers have done what many of us have talked about for a long time, i.e. observations of an ion tail at short time intervals over appreciable intervals of time. This procedure will certainly lead to important results in the future.

M. K. Wallis: The region of the solar wind disturbed by the cometary gas is surely much more limited than the extent of the $\mathrm{H}$-coma. For a comet of the size of Bennett, the H-coma appears thin to single protons outside $10^{4} \mathrm{~km}$. Taking heavy ions and general streaming into account, the distance may be increased to a few times $10^{5} \mathrm{~km}$, but far smaller than the $10^{7} \mathrm{~km}$ scale of the $\mathrm{H}$-coma or of the tail. This provides good reason for not devaluing the significance of comet tails as indicators of the solar wind.

J. C. Brandt: I think you should take your position and discuss it with the Munich group, and when you decide what the answer is, let me know. I tend to feel, however, that the flow is disturbed over a rather large distance. To what extent it is disturbed, I think, is still a valid question.

G. H. Herbig: Is it certain that such rapidly moving structures occur only in plasma tails, and never in dust tails? 


\section{DISCUSSION (Continued)}

J. C. Brandt: Yes, I think that is almost surely the case. There is fine structure that does occasionally show up in Type II tails, the so-called synchronic band structure, but I don't think it has any relation to this phenomenon whatsoever.

R. Lust: I have two questions. One concerns the ecliptic latitude of Comet Kohoutek when the tail showed the swan structure, the second with respect to a relationship with geomagnetic data.

J. C. Brandt: The comet was so close to Jupiter and Venus in the sky that it had to be at a low ecliptic latitude, and any relationship should show up. We searched our hearts out to find a solar event or a solar wind event. The spacecraft solar wind data is simply not availabie at the present time. We looked very hard at geomagnetic indices, and we found nothing we would like to have our names associated with at the present time.

R. Lust: Some velocities, especially those measured in the rays (wave structure) might not be velocities of material, but there are definitely structures (knots, condensations) for which material velocities of $10-100 \mathrm{~km} / \mathrm{sec}$ must be established.

J. C. Brandt: Obviously, there have to be motions of the tail. It is only when I see the type of structure, such as the two I specifically described today, that I become convinced that at least some of the structures must be wave patterns. Then I ask myself the question-let's be ridiculous, could they all be, in some not too exact way. The answer is, in terms of getting kinds of velocity variations that one finds-yes, indeed, they could possibly be. I think the best way to sort this matter out is with a spectrograph, and I think it would resolve it unambiguously. It is clear that if you have a more or less semi-cylindrical tail, that one of the ways to get the density to decrease is for the plasma to be accelerated down the tail, and if we ever get a good calibration point, we may be able to map that out fairly carefully.

D. A. Mendis: If we accept the value of $\mathrm{B}(\sim 100 \gamma)$, the question arises as to its origin. It is difficult to understand how the ambient interplanetary field establishes contact with the plasma in the tail, let alone be amplified. It may be suggested that the field is in fact intrinsic, being produced by the usual hydromagnetic conversion of turbulent energy to magnetic energy during the period of coma activity. Indeed, there is no difficulty energetically or with regards to the time scales for decay. 